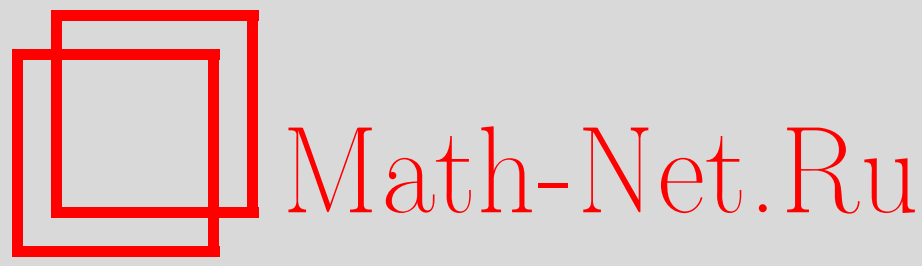

Е. И. Зеленов, Об операторах координаты и импульса в $p$-адической квантовой механике, ТМФ, 2010, том 164, номер 3, 426-434

DOI: https://doi.org/10.4213/tmf6553

Использование Общероссийского математического портала Math-Net.Ru подразумевает, что вы прочитали и согласны с пользовательским соглашением http://www.mathnet.ru/rus/agreement

Параметры загрузки:

IP : 54.162 .27 .143

26 апреля 2023 г., 13:20:55

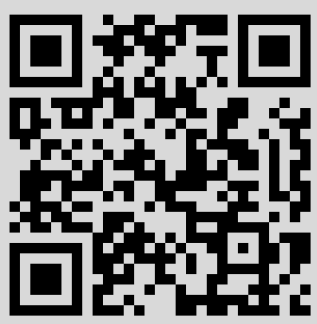




\title{
ОБ ОПЕРАТОРАХ КООРДИНАТЫ И ИМПУЛЬСА В $p$-АДИЧЕСКОЙ КВАНТОВОЙ МЕХАНИКЕ
}

\author{
Описываются операторы координаты и импульса в $p$-адической квантовой \\ механике на языке проекторнозначных мер.
}

Ключевые слова: p-адическая квантовая механика, спектральная мера, квантование Вейля.

\section{1. ВВЕДЕНИЕ}

В настоящей работе рассматривается вариант $p$-адической квантовой механики, в которой волновые функции - комплекснозначные функции $p$-адического аргумента. Естественная формулировка для такого подхода - квантование Вейля [1]. При этом вместо наблюдаемых (самосопряженных операторов) рассматриваются соответствующие однопараметрические унитарные группы операторов. Связь с привычной формулировкой в вещественном случае вполне естественна: от однопараметрических (сильно непрерывных) унитарных групп необходимо перейти к их самосопряженным генераторам, воспользовавшись теоремой Стоуна. В $p$-адическом случае такой переход невозможен.

На простейших, но содержательных примерах (операторы координаты и импульса) мы предлагаем процедуру построения самосопряженного оператора по соответствующей однопараметрической унитарной группе для случая $p$-адической квантовой механики. Оказывается, что спектр построенного таким образом оператора координаты (вернее, его ограниченного аналога) есть канторово множество.

$\mathrm{C}$ позиций настоящей статьи $p$-адическая квантовая механика по сути есть часть вещественной квантовой механики. Однако типичные операторы в $p$-адической квантовой механике имеют в качестве спектра множества типа канторовых.

\section{2. ПОСТАНОВКА ЗАДАЧИ}

Состояние классической системы есть точка в фазовом пространстве $F$. Будем рассматривать случай одномерной классической механики. В этом случае $F=$ $\mathbb{Q}_{p} \times \mathbb{Q}_{p}$ - двумерное векторное пространство над полем $p$-адических чисел. На пространстве $F$ задана симплектическая форма $B\left(z, z^{\prime}\right)=x y^{\prime}-x^{\prime} y, z=(x, y) \in F$,

*E-mail: evgeny.zelenov@gmail.com 
$z^{\prime}=\left(x^{\prime}, y^{\prime}\right) \in F$. Динамика классической системы задается семейством линейных преобразований $F$, сохраняющих симплектическую форму. В случае двумерного пространства $F$, который мы рассматриваем, эти преобразования образуют группу $S L_{2}\left(\mathbb{Q}_{p}\right)$.

ОПРЕДЕЛЕНИЕ 1. Классической р-адической (одномерной) системой назовем тройку $(F, B, G)$, где $F$ - двумерное векторное пространство над $\mathbb{Q}_{p}, B$ - невырожденная симплектическая форма на $F, G$ - подгруппа группы $S L_{2}\left(\mathbb{Q}_{p}\right)$, действующая на $F$ линейными преобразованиями.

В качестве подгруппы $G$ обычно выбирается однопараметрическая подгруппа, параметр при этом играет роль времени. Мы будем рассматривать произвольные подгруппы. Для $(x, y) \in F$ будем считать $x$ координатой классической частицы, а $y$ - импульсом классической частицы.

ОПРЕДЕЛЕНИЕ 2. Квантование предложенной классической системы задается четверкой $(U, V, H, \mathcal{U})$, где $H$ - комплексное гильбертово пространство, $U$ и $V$ - сильно непрерывные унитарные представления аддитивной группы поля $\mathbb{Q}_{p}$ в $H$, удовлетворяющие соотношению $U(x) V(y)=e^{i \pi\{x y\}_{p}} V(y) U(x)$ для всех $(x, y) \in F, \mathcal{U}$ - унитарное представление группы $G$ в $H$, удовлетворяющее соотношениям $\mathcal{U}(g) W(z)=$ $W(g z) \mathcal{U}(g)$, где $W(z)=U(x) V(y), z=(x, y) \in F$.

Состояние квантовой системы описывается волновой функцией - элементом пространства $H$ единичной нормы. Предложенное определение дает квантование в форме Вейля.

Приведем пример квантования (координатное представление). В качестве пространства $H$ выберем пространство $L_{2}\left(\mathbb{Q}_{p}\right)$. Операторы $U$ и $V$ зададим соотношениями

$$
\begin{aligned}
& (U(x) f)(u)=e^{i \pi\{x u\}_{p}} f(u), \\
& (V(y) f)(u)=f(u-y),
\end{aligned}
$$

$(x, y) \in F$. Далее будем использовать обозначение $e^{2 i \pi\{x\}_{p}}=\chi(x)$.

Представление $\mathcal{U}$ задается интегральными операторами, примеры для различных подгрупп $G$ можно найти в монографии [2].

В случае вещественной квантовой механики $U$ и $V$ образуют сильно непрерывные однопараметрические унитарные группы. Воспользовавшись теоремой Стоуна, можно перейти к самосопряженным генераторам этих групп и получить представление Шредингера, при котором операторы координаты и импульса суть замыкания операторов умножения и дифференцирования соответственно. В рассматриваемой нами $p$-адической квантовой механике нет аналога теоремы Стоуна, поскольку представление реализуется в комплексном пространстве, а параметр $p$-адический. Отсутствие генераторов создает определенные сложности при интерпретации $p$-адической квантовой механики.

Предлагается следующий выход из ситуации. Справедлива следующая теорема (см., например, [3]). 
Теорема 1. Всякое унитарное представление $g \rightarrow T_{g}$ коммутативной локально-компактной группы $G$ задается формулой

$$
T_{g}=\int \overline{\lambda(g)} d P(\lambda)
$$

где $P$ - некоторая проекторнозначная мера на группе характеров $\widehat{G}$ группь $G$.

Если $G=\mathbb{R}$, а $T_{g}$ - представление $\mathbb{R}$ в $L_{2}(\mathbb{R})$ операторами сдвига, указанная выше проекторнозначная мера есть проекторнозначная мера, соответствующая оператору дифференцирования (т.е. оператору импульса в представлении Шредингера).

Возвращаясь к p-адической квантовой механике, мы ставим задачу следующим образом: для представлений аддитивной группы поля $\mathbb{Q}_{p}$, задаваемых формулами (1), построить разложения вида (2). Соответствующие проекторнозначные меры будем называть проекторнозначными мерами операторов координаты и импульса.

\section{3. ОПЕРАТОРЫ КООРДИНАТЫ И ИМПУЛЬСА}

Справедлива следующая теорема.

Теорема 2. Проекторнозначная мера $Q$ для оператора координаты дается формулой

$$
\left(Q_{\Omega} f\right)(u)=h_{\Omega}(u) f(u),
$$

где $f \in L_{2}\left(\mathbb{Q}_{p}\right), \Omega$ - борелевское множество на $\mathbb{Q}_{p}, h_{\Omega}$ - характеристическая функиия множества $\Omega$.

Проекторнозначная мера для оператора импульса дается формулой

$$
\left(P_{\Omega} f\right)(u)=\left(\tilde{h}_{\Omega} * f\right)(u),
$$

где $\tilde{h}$ - преобразование Фуръе функиии $h, a *$ - оператор свертки.

ДокАЗАТЕЛьство теоремы элементарно. Поскольку пространство $\mathcal{D}$ локально-постоянных комплекснозначных функций на $\mathbb{Q}_{p}$ с компактным носителем плотно в $L_{2}\left(\mathbb{Q}_{p}\right)$, а любая функция из $\mathcal{D}$ есть конечная линейная комбинация характеристических функций шаров, доказательство достаточно провести для характеристической функции произвольного шара. Для шара $B \in \mathbb{Q}_{p}$ обозначим меру через $\mu(B)$. В качестве $f$ возьмем функцию $f=h_{B} / \sqrt{\mu(B)}$. Представим интеграл по проекторнозначной мере как предел сумм Римана. Для этого представим $\mathbb{Q}_{p}$ как объединение счетного числа непересекающихся шаров $B_{i}, i=1,2, \ldots$, одинакового радиуса:

$$
\begin{aligned}
\frac{1}{\mu(B)} & \int \chi(x u)\left(h_{B}, Q_{\Omega} h_{B}\right) d \mu(\Omega)= \\
& =\frac{1}{\mu(B)} \lim _{\mu\left(B_{i}\right) \rightarrow 0} \sum_{B_{i}} \chi\left(x u_{i}\right)\left(h_{B}, h_{B_{i}} h_{B}\right) \mu\left(B_{i}\right)= \\
& =\lim _{\mu\left(B_{i}\right) \rightarrow 0} \sum_{B_{i} \subset B} \chi\left(x u_{i}\right) \mu\left(B_{i}\right)=\int_{B} \chi(x u) d u=\frac{1}{\mu(B)}\left(h_{B}, \chi(x u) h_{B}\right) .
\end{aligned}
$$

Приведенные выкладки доказывают первое утверждение теоремы. 
Второе утверждение следует непосредственно из того факта, что проекторнозначные меры $Q_{\Omega}$ и $P_{\Omega}$ связаны преобразованием Фурье $F: P_{\Omega}=F^{-1} Q_{\Omega} F$ :

$$
P_{\Omega} f=F^{-1}\left[\tilde{h}_{\Omega} * F[f]\right]=h_{\Omega} f .
$$

Проекторнозначные меры $P_{\Omega}$ и $Q_{\Omega}$ не позволяют построить самосопряженные операторы импульса и координаты, однако они позволяют вычислять распределение вероятностей координаты и импульса. Кроме того, мы можем построить самосопряженные операторы, которые являются функциями от операторов координаты и импульса.

\section{4. ПРИМЕРЫ}

ПримеР 1. Пусть частица (в координатном представлении) находится в состоянии $f \in L_{2}\left(\mathbb{Q}_{p}\right), \Omega$ - борелевское множество в $\mathbb{Q}_{p}$. Вероятность того, что координата частицы лежит в области $\Omega$, дается формулой

$$
\mathcal{P}(x \in \Omega)=\int_{\Omega}|f(x)|^{2} d x .
$$

Пример 2. Через $B_{n}, n \in \mathbb{Z}$, обозначим шар радиуса $p^{n}$ с центром в нуле. Пусть состояние частицы описывается волновой функцией $f=p^{-n / 2} h_{B_{n}}$. Выберем целое $m$ таким образом, чтобы выполнялись неравенства $m \leqslant n \leqslant-m$. Найдем вероятность $\mathcal{P}\left(x \in B_{m}\right)$ того, что координата частицы принимает значения в шаре $B_{m}$. В соответствии с нашим подходом

$$
\mathcal{P}\left(x \in B_{m}\right)=p^{-n}\left(h_{B_{n}}, h_{B_{m}} h_{B_{n}}\right)=\frac{p^{m}}{p^{n}} .
$$

Вероятность $\mathcal{P}\left(y \in B_{m}\right)$ того, что импульс частицы принимает значение в шаре $B_{m}$, дается формулой

$$
\begin{aligned}
\mathcal{P}\left(y \in B_{m}\right) & =p^{-n}\left(h_{B_{n}}, \tilde{h}_{B_{m}} * h_{B_{n}}\right)= \\
& =p^{-n} \int h_{B_{n}}(\xi) p^{m} \int h_{B_{-m}}(\xi-\eta) h_{B_{n}}(\eta) d \xi d \eta=\frac{p^{n}}{p^{-m}} .
\end{aligned}
$$

Заметим, что если $n=0$ (т.е. частица находится в вакуумном состоянии, см. [2]) и $m=0$, то частица с вероятностью 1 находится в области фазового пространства $\mathbb{Z}_{p} \times \mathbb{Z}_{p}$

Как уже отмечалось, предлагаемый подход не дает возможности построить операторы координаты и импульса, однако позволяет строить функции от таких операторов.

\section{5. p-АДИЧЕСКАЯ СПЕКТРАЛЬНАЯ МЕРА}

Предлагаемый подход позволяет несколько по-другому посмотреть на $p$-адическую механику и ее связь с вещественной квантовой механикой.

Мы рассматривали проекторнозначные меры на $\mathbb{Q}_{p}$, при этом операторы проектирования действовали на пространстве $L_{2}\left(\mathbb{Q}_{p}\right)$. Однако выбор пространства непринципиален. Легко построить проекторнозначную меру на $\mathbb{Q}_{p}$, при этом проекторы будут действовать в $L_{2}(\mathbb{R})$. Можно воспользоваться изоморфизмом сепарабельных 
гильбертовых пространств, но мы построим явный пример. Для простоты рассуждений приведем пример для случая $p=2$, для произвольного $p$ рассуждения аналогичны. В пространстве $L_{2}(\mathbb{R})$ выберем ортонормированный базис $\left\{\phi_{n}, n \in \mathbb{N}\right\}$. Разложим пространство в ортогональную прямую сумму пространств $H_{0}$ и $H_{1}$, где $H_{0}$ и $H_{1}$ натянуты на векторы с четными и нечетными номерами соответственно. Проекторы на $H_{0}$ и $H_{1}$ обозначим через $P_{0}$ и $P_{1}$. Проектору $P_{0}$ поставим в соответствие единичный шар $B_{0}$ в $\mathbb{Q}_{2}$ с центром в нуле, а проектору $P_{1}$ - дополнение в $\mathbb{Q}_{2}$ к этому шару. Теперь пространство $H_{0}$ разложим в ортогональную прямую сумму подпространств $H_{00}$ и $H_{01}$, натянутых на векторы, номера которых при делении на 4 имеют остатки 0 и 2 соответственно. Шар единичного радиуса в $\mathbb{Q}_{2}$ есть объединение двух непересекающихся шаров $B_{00}$ и $B_{01}$ радиуса $1 / 2$. Этим шарам поставим в соответствие проекторы $P_{00}$ и $P_{01}$ на подпространства $H_{00}$ и $H_{01}$. Аналогично поступим с пространством $H_{1}$, рассмотрев подпространства, натянутые на векторы с номерами, имеющими при делении на 4 остатки 1 и 3 соответственно. Проектору $P_{10}$ поставим в соответствие единичный шар $B_{10} \subset \mathbb{Q}_{2} \backslash B_{0}$, а проектору $P_{11}$ - дополнение в $\mathbb{Q}_{2}$ к множеству $B_{0} \cup B_{10}$. Легко увидеть, что продолжая эту процедуру, каждому шару в $\mathbb{Q}_{2}$ мы поставим в соответствие проектор в $L_{2}(\mathbb{R})$. Это соответствие задает требуемую проекторнозначную меру.

Далее заметим, что поскольку $\mathbb{Q}_{p}$ и $\mathbb{R}$ - полные метрические пространства одинаковой мощности, то они изоморфны как борелевские пространства, через $\beta: \mathbb{Q}_{p} \rightarrow \mathbb{R}$ обозначим этот изоморфизм. $\mathrm{C}$ помощью $\beta$ меру на $\mathbb{Q}_{p}$ можно перенести на $\mathbb{R}$, $\nu_{\mathbb{R}}(\Omega)=\mu_{\mathbb{Q}_{p}}\left(\beta^{-1} \Omega\right)$, где $\Omega$ - борелевское подмножество $\mathbb{R}$, а $\mu_{\mathbb{Q}_{p}}$ - борелевская мера на $\mathbb{Q}_{p}$. Таким же образом построенная проекторнозначная мера на $\mathbb{Q}_{p}$ задает некоторую проекторнозначную меру на $\mathbb{R}$. Эта мера позволяет строить самосопряженные операторы (наблюдаемые) вещественной квантовой механики, соответствующие наблюдаемым в $p$-адической квантовой механике. В следующем разделе мы уточним предложенную идею.

\section{6. СПЕКТР ОПЕРАТОРА КООРДИНАТЫ}

Рассмотрим более подробно оператор p-адической координаты. Для простоты ограничимся случаем, когда волновые функции имеют носитель в единичном шаре в $\mathbb{Q}_{p} . \mathrm{B}$ пространстве $H_{p}=L_{2}\left(\mathbb{Z}_{p}\right)$ определим проекторнозначную меру $Q$, как в теореме 2:

$$
\left(Q_{\Omega} \psi\right)(u)=h_{\Omega}(u) \psi(u), \quad \psi \in L_{2}\left(\mathbb{Z}_{p}\right),
$$

где $\Omega$ - борелевское множество в $\mathbb{Z}_{p}$.

Как уже отмечалось, построенная проекторнозначная мера $Q$ не позволяет получить оператор $p$-адической координаты $\mathcal{Q}$ путем простого интегрирования функции $f(x)=x, x \in \mathbb{Z}_{p}$, по этой мере, поскольку $f$ принимает значения в поле $p$-адических чисел, однако мы можем построить аналог функций от оператора координаты.

Действительно, пусть $F: \mathbb{Z}_{p} \rightarrow \mathbb{C}$ - измеримая по Борелю ограниченная комплекснозначная функция. Тогда

$$
F(\mathcal{Q})=\int_{\mathbb{Z}_{p}} F(\lambda) d Q(\lambda)
$$


задает ограниченный оператор на $H_{p}$. Если функция $F$ принимает вещественные значения, то оператор $F(\mathcal{Q})$ самосопряженный. Легко видеть, что оператор $F(\mathcal{Q})$ имеет вид

$$
(F(\mathcal{Q}) \psi)(x)=F(x) \psi(x), \quad \psi \in H_{p} .
$$

Другими словами, это оператор умножения на функцию $F$.

Рассмотрим следующий пример.

Пример 3. Пусть $x=x_{0}+x_{1} p+\cdots+x_{n} p^{n}+\cdots-$ каноническое разложение целого $p$-адического числа $x, N$ - натуральное число. Рассмотрим функцию $F_{N}(x)=$ $x_{0}+x_{1}+\cdots+x_{N-1} p^{N-1}$. Функция $F_{N}$ непрерывна, локально-постоянна (постоянна на каждом шаре радиуса $p^{-N}$ ) и принимает значения в множестве целых чисел $\left\{0,1,2, \ldots, p^{N}-1\right\}$. Справедливо равенство

$$
\lim _{N \rightarrow \infty}\left|F_{N}(x)-x\right|_{p}=0,
$$

в этом смысле функция $F_{N}$ аппроксимирует линейную функцию.

Оператор $F_{N}(\mathcal{Q})$ обладает следующими свойствами:

а) $F_{N}(\mathcal{Q})$ - самосопряженный оператор;

б) собственные значения оператора $F_{N}(\mathcal{Q})$ пробегают множество целых чисел от 0 до $p^{N}-1$;

в) все собственные значения оператора $F_{N}(\mathcal{Q})$ имеют бесконечную кратность.

Следующим естественным шагом в построении $p$-адического оператора координаты является рассмотрение $C^{*}$-алгебры операторов, порожденной семейством проекторов $\left\{Q_{\Omega}\right\}$, где $\Omega$ пробегает семейство борелевских подмножеств $\mathbb{Z}_{p}$. Обозначим эту алгебру через $A_{\mathcal{Q}}$. Заметим, что в вещественном случае эта алгебра есть алгебра непрерывных функций на спектре оператора координаты.

Справедлива следующая теорема.

Теорема 3. Алгебра $A_{\mathcal{Q}}$ изоморфна алгебре $C\left(\mathbb{Z}_{p}\right)$ непрерывных функиий на единичном шаре в $\mathbb{Z}_{p}$.

ДокАзАТЕЛьство следует из того факта, что любая функция из $C\left(\mathbb{Z}_{p}\right)$ есть равномерный предел конечных линейных комбинаций характеристических функций шаров в $\mathbb{Z}_{p}$.

Заметим, что алгебра $C\left(\mathbb{Z}_{p}\right)$ изоморфна алгебре $C(\mathcal{C})$ непрерывных функций на канторовом множестве $\mathcal{C}$ (поскольку $\mathbb{Z}_{p}$ гомеоморфно $\mathcal{C}$ ).

Гомеоморфизм $\alpha: \mathbb{Z}_{p} \rightarrow \mathcal{C}$ строится следующим образом. Пусть $a=a_{0}+a_{1} p+\cdots$ $\cdots+a_{n} p^{n}+\cdots-$ целое $p$-адическое число. Определим

$$
\alpha(a)=\frac{p}{p-1}\left(a_{0}+\frac{a_{1}}{p+1}+\cdots+\frac{a_{n}}{(p+1)^{n}}+\cdots\right) .
$$

Видно, что $\alpha$ есть непрерывное вложение $\mathbb{Z}_{p}$ в отрезок $[0,1]$ вещественной прямой. Образом этого отображения являются вещественные числа, у которых в разложении по обратным степеням числа $p+1$ отсутствует число $p$, т.е. канторово множество, которое мы обозначили через $\mathcal{C}$ (см., например, [4]). 
Построенное отображение $\alpha$ позволяет перенести меру Хаара (и, соответственно, проекторнозначную меру $Q)$ с $\mathbb{Z}_{p}$ на отрезок $[0,1]$. Действительно, пусть $\mu_{p}-$ мера Хаара на $\mathbb{Z}_{p}$. Через $\mu_{p}^{\alpha}$ обозначим меру на $[0,1]$, которая задается следующим образом:

$$
\mu_{p}^{\alpha}([a, b])=\mu_{p}\left(\alpha^{-1}(\mathcal{C} \cap[a, b])\right) .
$$

Построенная мера $\mu_{p}^{\alpha}$ на отрезке $[0,1]$ непрерывна и сингулярна по отношению к мере Лебега, носитель этой меры - канторово множество $\mathcal{C}$. Аналогичным образом определим проекторнозначную меру $Q^{\alpha}$ на отрезке $[0,1]$ :

$$
Q^{\alpha}([a, b])=Q\left(\alpha^{-1}(\mathcal{C} \cap[a, b])\right) .
$$

Построенная мера $Q^{\alpha}$ делает естественным следующее определение.

ОПРЕДЕЛЕНИЕ 3. Оператором $\mathcal{Q}^{\alpha}$ координатъ в р-адической квантовой механике (или просто р-адическим оператором координаты) назовем следующий оператор:

$$
\mathcal{Q}^{\alpha}=\int_{0}^{1} \lambda d Q^{\alpha}(\lambda)
$$

Оператор $\mathcal{Q}^{\alpha}$ выглядит довольно сложно в вещественном представлении. В действительности это весьма простой оператор.

Лемма 1. -Адический оператор координатъ $\mathcal{Q}^{\alpha}$ есть оператор умножения на функиию $\alpha$.

ДокАзАТЕЛьство. В силу построения меры $Q^{\alpha}$ справедлива следующая формула:

$$
\mathcal{Q}^{\alpha}=\int_{0}^{1} \lambda d Q^{\alpha}(\lambda)=\int_{\mathbb{Z}_{p}} \alpha(\lambda) d Q(\lambda),
$$

а последний оператор есть оператор умножения на $\alpha$ в силу свойств проекторнозначной меры $Q$.

Как легко заметить, спектр оператора $\mathcal{Q}^{\alpha}$ есть канторово множество на отрезке $[0,1]$.

Из леммы 1 вытекает довольно любопытное следствие.

ТЕОРема 4. Любую непрерывную комплекснозначную функиию на $\mathbb{Z}_{p}$ можно равномерно приблизить полиномами от функции $\alpha$.

Действительно, как следует из леммы 1 , функция $\alpha$ порождает алгебру $C\left(\mathbb{Z}_{p}\right)$.

Заметим, что спектр построенного оператора $\mathcal{Q}^{\alpha}$ есть канторово множество $\mathcal{C}$.

В дальнейшем удобно будет использовать в качестве отображения $\alpha$ другую функцию. Пусть $d$ - вещественное число, $0<d<1$. Для целого $p$-адического числа $a=a_{0}+a_{1} p+\cdots+a_{n} p^{n}+\cdots$ определим вещественнозначную функцию $\alpha_{d}$ по формуле

$$
\alpha_{d}(a)=\frac{p^{1 / d}-1}{(p-1) p^{1 / d}}\left(a_{0}+\frac{a_{1}}{p^{1 / d}}+\cdots+\frac{a_{n}}{p^{n / d}}+\cdots\right) .
$$

Как было показано в работе [5], отображение $\alpha_{d}$ - непрерывное вложение $\mathbb{Z}_{p}$ в отрезок $[0,1]$, образом этого отображения является канторово множество $\mathcal{C}_{d}$, хаусдорфова размерность этого множества равна $d$. Все построения, сделанные ранее для 
отображения $\alpha$, верны и для $\alpha_{d}$. Кроме того, легко заметить, что $\alpha$ совпадает с $\alpha_{d}$ при $d=1 / \log _{p}(1+p)$.

Для построения оператора $p$-адической координаты мы использовали непрерывное вложение $\mathbb{Z}_{p}$ в отрезок $[0,1]$. Посмотрим теперь, какой оператор мы получим, если вместо непрерывного вложения будем использовать борелевский изоморфизм $\mathbb{Z}_{p}$ и $[0,1]$. В некотором смысле, построенный ниже изоморфизм будет пределом отображений $\alpha_{d}$ при $d \rightarrow 1$.

Обозначим через $\mathcal{Z}_{p}$ множество целых $p$-адических чисел с выколотыми целыми рациональными точками (т.е. точками с конечными каноническими разложениями). Множество $\mathcal{Z}_{p}$ локально компактно и оснащено естественной борелевской структурой как подмножество $\mathbb{Z}_{p}$.

Определим отображение $\beta: \mathcal{Z}_{p} \rightarrow[0,1]$ по формуле

$$
\beta(a)=\frac{1}{p}\left(a_{0}+a_{1} p^{-1}+a_{2} p^{-2}+\cdots+a_{n} p^{-n}+\cdots\right),
$$

где $a=a_{0}+a_{1} p+\cdots+a_{n} p^{n}+\cdots-$ каноническое разложение $a \in \mathcal{Z}_{p}$.

Обратное отображение $\beta^{-1}:[0,1] \rightarrow \mathcal{Z}_{p}$ определяется очевидным образом.

Справедлива следующая очевидная лемма.

ЛЕмма 2. Отображение $\beta$ отображает $\mathcal{Z}_{p}$ на $[0,1]$ взаимно однозначно. Отображение $\beta$ непрерывно, обратное отображение $\beta^{-1}$ разрывно в счетном числе точек.

Из леммы 2 вытекает, что $\beta$ является борелевским изоморфизмом $\mathcal{Z}_{p}$ и $[0,1]$ (более того, $\beta$ является обобщенным гомеоморфизмом класса $(0,1)$, см. [4]).

$\mathrm{C}$ помощью изоморфизма $\beta$ определим меру $\mu$ на отрезке $[0,1]$ по формуле $\mu(\Omega)=$ $\mu_{p}\left(\beta^{-1}(\Omega)\right)$, где $\Omega$ - борелевское подмножество отрезка $[0,1]$, а $\mu_{p}$ - мера на $\mathcal{Z}_{p}$, индуцированная мерой Хаара на $\mathbb{Z}_{p}$.

Непосредственно из построения меры $\mu$ вытекает следующая теорема.

ТеОрема 5. Мера н абсолютно непреръвна относителъно меры Лебега.

Отображение $\beta$ очевидным образом продолжается до непрерывного отображения $\mathbb{Z}_{p} \rightarrow[0,1]$, которое будем обозначать тем же символом $\beta$. Аналогично оператору $\mathcal{Q}^{\alpha}$ (см. определение 3 ) построим оператор $\mathcal{Q}^{\beta}$, который есть оператор умножения на функцию $\beta$. Из теоремы 5 непосредственно следует, что спектр оператора $\mathcal{Q}^{\beta}$ есть отрезок $[0,1]$. В отличие от оператора $\mathcal{Q}^{\alpha}$, оператор $\mathcal{Q}^{\beta}$ не порождает алгебру $C\left(\mathbb{Z}_{p}\right)$ непрерывных функций на $\mathbb{Z}_{p}$. Как легко заметить, оператор $\mathcal{Q}^{\beta}$ порождает подалгебру $B$ в $C\left(\mathbb{Z}_{p}\right)$, состоящую из функций, обладающих следующим свойством. Пусть $a=a_{0}+a_{1} p+\cdots+a_{n} p^{n}$ - целое рациональное число. Непрерывная функция $f$ лежит в алгебре $B$ тогда и только тогда, когда выполнено равенство $f(a)=f\left(a-p^{n+1}\right)$ для всех целых рациональных $a$. Отметим также, что алгебра $B$ изоморфна алгебре $C([0,1])$ непрерывных функций на отрезке $[0,1]$. Поэтому "правильным" оператором координаты следует считать оператор $\mathcal{Q}^{\alpha}$. 


\section{7. ЗАКЛЮЧИТЕЛЬНЫЕ ЗАМЕЧАНИЯ}

Как отмечалось в начале статьи, одна из целей данной работы - попытка глубже понять связь между вещественной и р-адической квантовыми механиками. С позиций настоящей статьи $p$-адическая квантовая механика по сути есть часть вещественной квантовой механики. Однако “типичные" операторы в $p$-адической квантовой механике имеют в качестве спектра множества типа канторовых, а "типичные" меры непрерывны и сингулярны относительно меры Лебега. В этом смысле p-адическая квантовая механика может выступать инструментом описания некоторого класса сложных квантово-механических систем. Заметим, что эта идея высказывалась в ранних работах по р-адической математической физике (библиографию см. в [2], [6]).

Благодарности. Автор выражает благодарность И. В. Воловичу за многочисленные плодотворные обсуждения идей и результатов работы. Работа финансировалась Программой поддержки ведущих научных школ (грант НШ-3224.2008.1).

\section{Список литературы}

[1] В. С. Владимиров, И. И. Волович, Докл. АН СССР, 302:2 (1988), 320-323.

[2] В. С. Владимиров, И. В. Волович, Е.И. Зеленов, р-Адический анализ и математическая физика, Наука, М., 1994.

[3] М. А. Наймарк, Нормированнье кольща, Наука, М., 1968.

[4] К. Куратовский, Топология. т. 1, 2, Мир, М., 1966, 1969.

[5] S. Fischenko, E. Zelenov, "p-Adic models of turbulence", p-Adic Mathematical Physcis, AIP Conf. Proc., 286, eds. A. Yu. Khrennikov, Z. Rakic, I. V. Volovich, AIP, New York, 2006, 174-191.

[6] B. Dragovich, A. Yu. Khrennikov, S. V. Kozyrev, I. V. Volovich, Ultrametric Anal. Appl., 1:1 (2009), 1-17, arXiv: 0904.4205. 